\title{
Pick it up with light! An advanced summer program for secondary school students
}

Manoj Mathew, S. Kumar, Alejandra Valencia, Giorgio Volpe, Giovanni Volpe, et al.

Manoj Mathew, S. Chaitanya Kumar, Alejandra Valencia, Giorgio Volpe, Giovanni Volpe, Silvia Carrasco, "Pick it up with light! An advanced summer program for secondary school students," Proc. SPIE 9289, 12th Education and Training in Optics and Photonics Conference, 92892U (17 July 2014); doi: $10.1117 / 12.2070771$

SPIE Event: 12th Education and Training in Optics and Photonics Conference, 2013, Porto, Portugal 


\title{
"Pick it up with light!"- An Advanced Summer Program for Secondary School Students
}

\author{
Manoj Mathew, ${ }^{1}$ S. Chaitanya Kumar, ${ }^{1, *}$ Alejandra Valencia, ${ }^{1,2}$ Giorgio Volpe, ${ }^{1}$ Giovanni Volpe, ${ }^{1}$ \\ and Silvia Carrasco ${ }^{1}$ \\ ${ }^{1}$ ICFO-Institut de Ciencies Fotoniques, Mediterranean Technology Park, 08860 Castelldefels, \\ Barcelona, Spain \\ ${ }^{2}$ Quantum Optics Laboratory, Universidad de los Andes, A.A. 4976, Bogotá D.C., Colombia
}

\begin{abstract}
A project to introduce secondary school students to statistical physics and biophotonics by means of an optical tweezers is presented. Interestingly, the project is completely experimental and no advanced calculus or physics knowledge is necessary. The project starts from the construction of the optical tweezers itself and therefore is also useful to introduce basic concepts of optics.

Keywords: Optics education, Optics experimental demonstrations, Educational outreach, Fascination of light, Biophotonics, Optical tweezers.
\end{abstract}

\section{INTRODUCTION}

There is usually a huge disparity between the levels at which science, and physics in particular, is taught at school and at university. Even students who do really well at school very often struggle to cope with undergraduate physics programs. A way to approach this issue is to familiarize the higher-secondary-school students with at least some aspects of the subjects that are going to be treated at university. This, however, sets the challenge of introducing advanced concepts in a very simple language, because the students do not know advanced mathematical tools such as calculus yet.

Here, we report on a hands-on approach to introduce concepts of optics, statistical physics, and biophotonics to higher-secondary-school students using an economic and relatively easy-to-build optical tweezers. The idea derives from the author's experience ${ }^{1,2}$ in developing and guiding a project named Pick it up with light!, which was conceived for a two-week summer workshop directed to Spanish higher-secondary-school students ${ }^{3}$. The students were involved in all the steps that led to the construction of the optical tweezers, acquiring the fundamental concepts of optics and the basic experimental skills required to build optic al setups. Furthermore, they used it to perform some simple, yet fundamental, experiments in statistical physics and biophotonics by trapping micrometer-sized particles and individual living biological cells.

Among the various possible subjects for the experiments, statistical physics and biophotonics were chosen for some specific reasons. Statistical physics is, indeed, one of the pillars of modern physics; however, its connection with everyday experience is often hidden by a highly abstract presentation. An optically trapped Brownian particle constitutes an ideal system for establishing such connection: the students can directly manipulate a single microscopic particle and have a first-hand experience of the interplay between deterministic forces - the trapping potential - and noise - the Brownian motion - a topic that is at the foundation of statistical physics. From this standpoint, it is possible to introduce the students in a natural and straightforward way to very abstract concepts, e.g., the fractal nature of Brownian motion ${ }^{4}$ and the constructive role of noise in phenomena such as stochastic resonance ${ }^{5}$, Kramer transitions ${ }^{6}$, and stochastic resonant damping ${ }^{7}$. Interdisciplinary sciences are the key to solve complex problems. Unfortunately, natural

*chaitanya.suddapalli@icfo.es

12th Education and Training in Optics and Photonics Conference, edited by

Manuel F. P. C. Martins Costa, Mourad Zghal, Proc. of SPIE Vol. 9289, 92892

(C) 2014 SPIE, OSA, IEEE, ICO · doi: 10.1117/12.2070771

Proc. of SPIE Vol. 9289 92892U-1 
sciences are often taught setting a clear separation between the various subdisciplines, a practice that is as established as harmful. Biophotonics constitutes an excellent example of an alternative paradigm. It is a highly interdisciplinary science that combines concepts of physics and biology with an eye for technological applications. The possibility of trapping individual cells can be used to introduce topics related to biology and complement in this way the existing higher-secondary-school programs. In the summer camp, for example, the students trapped a single yeast cell (Saccharomyces cerevisiae), which is a widely used organism for fundamental studies of cell processes ${ }^{8}$, readily available and non-toxic; they studied its life-cycle, which usually lasts a few hours for an optically trapped yeast cell ${ }^{9,10}$.

While some previous works ${ }^{11-13}$ have reported the use of optical tweezers as a teaching aid for introducing statistical physics and biophotonics, these efforts were directed towards undergraduate students that already have some calculus background. Our innovative aim is to demonstrate the possibility of introducing these concepts to higher-secondary school students that do not possess either the mathematics or the physics background that the university students have. In this paper, we give a comprehensive description of the program we devised and of the student's reactions to it. These sometimes included unexpected questions and doubts from the students, which we discuss. We hope this work can inspire similar efforts that contribute to the increase of scientific literacy.

\section{BUILDING AND UNDERSTANDING AN OPTICAL TWEEZERS SETUP}

The building of the optical tweezers is done in two steps: (1) a microscope is constructed; (2) a laser beam is coupled into the microscope objective to generate the optical tweezers. As the construction of the setup progresses, the necessary theory is explained with the help of simple demonstrations based on basic physics, mathematics, geometrical optics diagrams, and common sense. By our experience, most of the concepts are grasped by the students as a result of their own experience in building the setup.

\subsection{The microscope \& basic optics concepts}
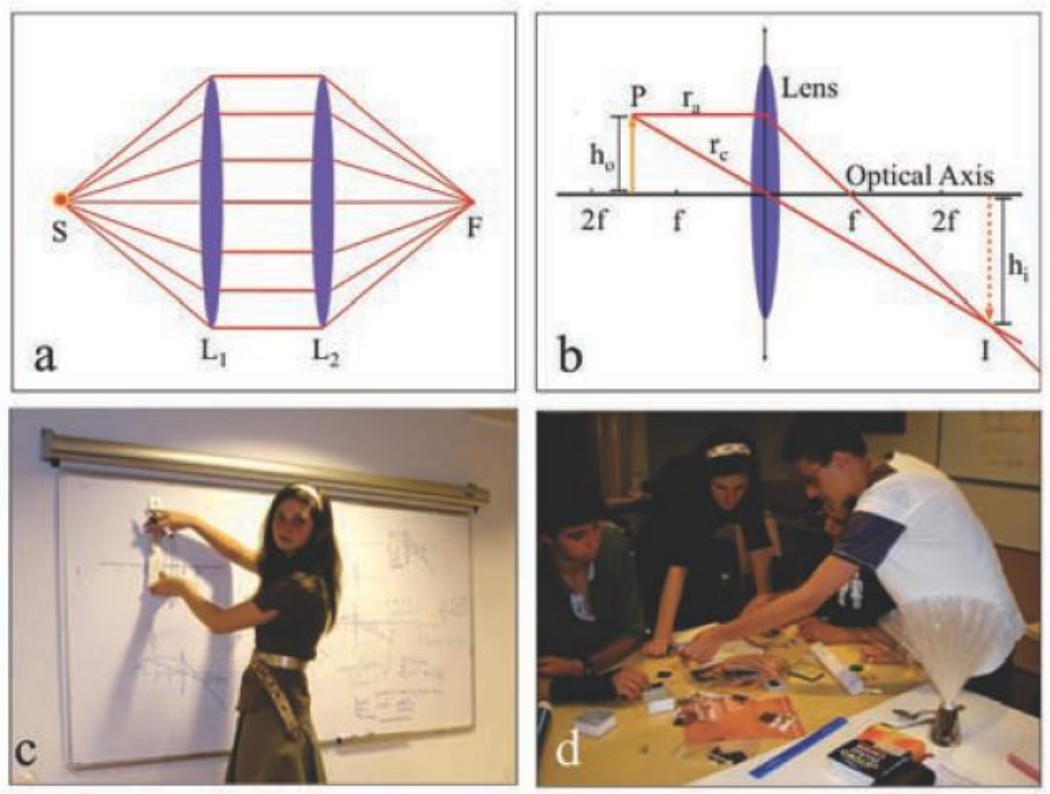

FIG. 1: Introduction to ray optics. (a) A ray-optics model with a point light-source (S) emitting a diverging light-beam, a lens (L1) collimating it and a second lens (L2) focusing it into a point (F). (b) Ray-optics model of the image formation process. (c) A student drawing ray-optics diagrams. (d) Students double-checking the predictions of the ray-diagrams with simple experiments. 
The students did not have any prior knowledge of optics. We started introducing to them the concept of a light-ray by appealing on the familiar fact that light propagates in a straight line. We could then introduce the concepts of a point light-source (S) and the working principle of a lens $\left(\mathrm{L}_{1}\right.$ and $\left.\mathrm{L}_{2}\right)$ [Fig. 1(a)]. The students could grasp the difference between a diverging, collimated, and converging light-beam, by drawing ray optics diagrams themselves.

Once the students were familiarized with these principles, it was possible to introduce them the image formation process and the microscope working principle. Fig. 1(b) presents the ray diagram used to explain how a convex lens with focal distance $\mathrm{f}$ forms an image of an object kept between $\mathrm{f}$ and $2 \mathrm{f}$. From each point $\mathrm{P}$ of the object, two light rays are drawn: one that travels parallel to the lens optic axis $\left(r_{a}\right)$ and another that passes through its center $\left(r_{c}\right)$. The ray $r_{a}$ is bent by the lens such that it passes through the focus, while $r_{c}$ travels through the lens undeflected. The image I of $\mathrm{P}$ is formed where $r_{a}$ and $r_{c}$ meet. It is easily understood from the ray diagram that an inverted and magnified image of the object is formed on the opposite side of the lens at a distance between $2 \mathrm{f}$ and infinity. The students were encouraged to draw similar ray diagrams with the object placed at different distances from the center of the lens [Fig.1(c)]. In addition, they could experimentally verify the prediction of their diagrams using a convex lens, an object, a light source, and a screen [Fig. 1(d)].

Once the students understood how a single lens forms images, it was easy for them to figure out how in a microscope the combination of two lenses can magnify an image. The first lens (the objective) collects and collimates the light from each point of the sample, and the second lens focuses all the rays on a screen where the image can be seen, in a scheme similar to the one in Fig. 1(a). The screen can obviously be a CCD camera that further projects the image on a monitor.

Once the theory was understood, the students started constructing a platform where the microscope was going to be mounted. This simple task introduced them to the most practical aspects of experimental optics. The platform was built mounting an optical breadboard $(50 \times 20 \mathrm{~cm}$, Thorlabs, MB3030/M) on a portable optical table $(90 \times 60 \mathrm{~cm}$, Thorlabs, PBH51506) using four posts $(25 \mathrm{~cm}$, Thorlabs, P250/M) [Fig. 2(a)]. The students mounted a xy-translation stage (Newport, M-406) on top of the platform to act as a movable sample holder [Fig. 2(b)]. A square piece of $5 \times 5 \mathrm{~cm}$ had been cut away from the breadboard center in advance to facilitate the insertion of the microscope objective from below the platform. The microscope objective (100x, 1.3NA, oil immersion, Edmund Optics, Nr. 38344) was mounted on a ztranslation stage (Thorlabs, PT1A/M) to allow vertical movement with respect to the sample plane [Fig. 2(b)]. The sample was illuminated by a LED light-source (Thorlabs, MGLED) collimated by a condenser objective lens (40x, 0.7NA, Edmund Optics, Nr. 38343) mounted on a z-translation stage (Thorlabs, PT1A/M) and a xy-translational stage (Thorlabs, ST1XY-D/M) [Fig. 2(c)]. The illumination light was collected by the objective, reflected by a dichroic hot mirror (cutoff wavelength $580 \mathrm{~nm}$, Thorlabs, FM02), and focused by a $200 \mathrm{~mm}$ lens onto a CCD camera (Rister, CCD 3035) [Fig. 2(d)]. The magnified image can then be seen on a LCD screen (Amstrad, LCT 1044).

Once the students managed to mount the microscope, they were encouraged to collect objects they could observe with the microscope. They observed leaves, hair, skin, insects, water from a nearby stream, paper, textile fibers at 20X, 40X, and 60X magnifications. This was an important point of the learning process because they were now able to use autonomously the microscope. They were even proud of independently correcting problems arising due to slight misalignment of some components.
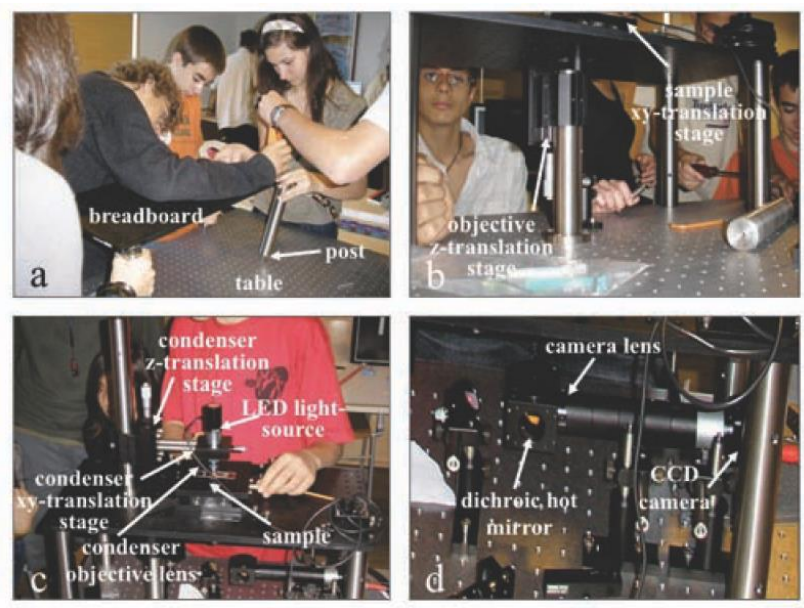

FIG. 2: Construction of the microscope: (a) the microscope platform; (b) the sample xy-translation stage; (c) illumination LED and condenser lens; (d) imaging. 


\subsection{The optical tweezers \& the trapping process}

The basic working principle behind optical tweezers relies on light exerting a force on a microscopic dielectric sphere ${ }^{14}$. A deep understanding of this concept requires knowledge of electromagnetism. However, by simple examples, analogies, and ray diagrams it is possible to make the students grasp the physics of the trapping process. The idea of light exerting a force is not an intuitive one. Quoting J. H. Poynting's presidential address to the British Physical Society in 1905 as cited in Ref.15, "a very short experience in attempting to measure these forces is sufficient to make one realize their extreme minuteness - a minuteness which appears to put them beyond consideration in terrestrial affairs." And this is true for virtually all our everyday experience. Hence, first of all the students must be convinced that light can indeed exert forces. They were, therefore, reminded of a phenomenon they studied at school: The fact that comet tails point away from the sun, a phenomenon already described in 1619 by Kepler's De Cometis. As a comet approaches the sun, the solar radiation vaporizes its volatile materials. An ionized gas tail is pushed by the solar wind and a dust tail, which is made of neutral particles, is pushed away from the sun by the radiation pressure. This fact convinced them of the existence of light-induced forces. At this point, the students were reminded of the fact that a force corresponds to the change in the momentum of a body. This was relatively easy because such concept is covered in higher-secondaryschool programs. However, it was useful to point out that the momentum is the product of mass and velocity and, therefore, a change in velocity results in a force. Everything was now set to explain how an optical tweezers works. From our experience, this is the toughest point for the student to grasp. We went back to the ray-optics diagram, which the students enjoyed and understood, and we examined what happens to a light-ray when it travels through a dielectric sphere [Fig.3(a)]. This led naturally to consider the phenomena of reflection and refraction at an interface, which the students could experience first-hand using a laser pointer with some plastic transparent geometrical shapes. An incoming light-ray entering a dielectric sphere changes its propagation direction because of refraction and, therefore, its momentum changes. This induces a recoil force acting on the sphere (the students were already familiar with Newton's third law of actio-reactio). This force (dark gray arrow in Fig.3(a)), however, pushes the sphere away from the light and, hence, cannot trap it.

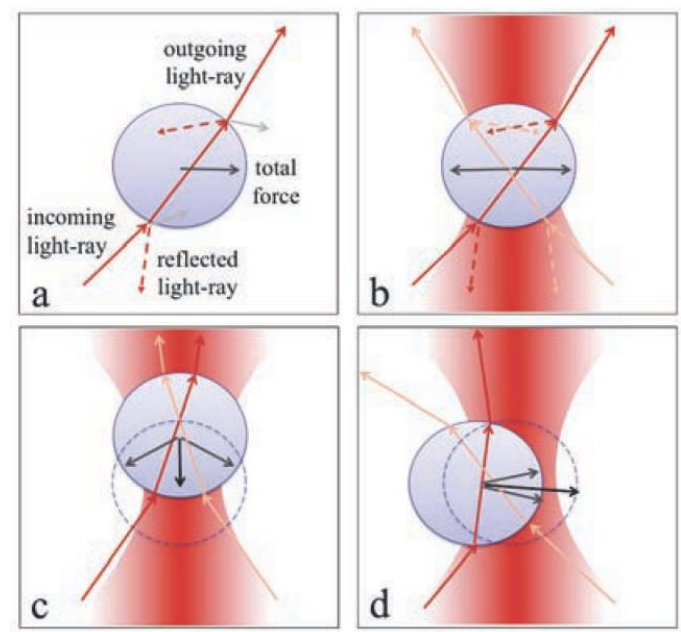

FIG. 3: Optical forces on a dielectric sphere as they can be understood with a ray diagram. (a) A light-ray (red) exert a force (dark gray) arising from its refraction and reflection. (b) The forces (dark gray) due to two light-rays (red and orange) acting on a sphere compensate each-other at the equilibrium position. (c) Restoring force on a axially displaced sphere. (d) Restoring force on a laterally displaced sphere.

To trap an object it is necessary to use a set of light-rays coming from different directions ${ }^{16}$. The students drew raydiagrams with two light-rays coming from opposite sides of the dielectric sphere and in this way they noticed that only using two rays coming with a very high angle they could indeed trap the particle [Fig. 3(b)]. After that, the students were encouraged to explore what happens when the sphere is displaced both axially [Fig. 3(c)] and laterally [Fig. 3(d)] with respect to the focus. In this cases the total force (black arrow) pushes the particle towards the optical trap center arises. An applet ${ }^{17}$ was very useful in developing the students' intuition of optical forces. 
Once the theory of optical trapping was clear to the students, they started modifying the microscope into an optical tweezers. It was at this point clear to them that they needed to couple a laser beam to the objective and that the objective would do the work of bending the light-rays in order to create an optical trap. The laser beam from a $630 \mathrm{~nm}$ diode laser

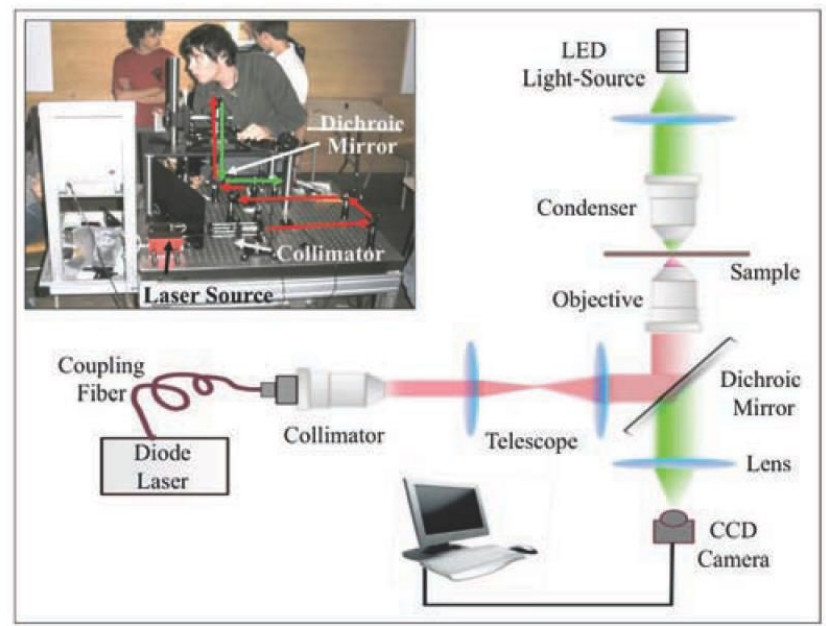

FIG. 4: Schematic of the optical tweezers setup. Inset: Light-path of the trapping beam (red) and of the illumination light (green).

with an output fiber coupler (OZ Optics, NEW-OZ-2000-635-4/125-S-40-3A-3A-1-20) was collimated with the help of an objective lens (10x, Edmund Optics, 38342) and directed into the microscope objective [red light-path in the inset of Fig. 4]. The hot dichroic mirror separated the red trapping light from the green light used to illuminate the sample. Finally, the whole setup was covered by an enclosure to isolate it from ambient stray light and to comply with lasersafety issues. The schematic of the whole setup is shown in of Fig. 4.

In order to verify that the optical tweezers was indeed working, the students performed various simple experiments. They optically trapped polystyrene beads (diameter $1 \mu \mathrm{m}$, Bang Labs) and silica beads (diameter $3 \mu \mathrm{m}$, Bang Labs). They verified that the particles were effectively trapped by moving the sample on the translation stage. Finally, the students were encouraged to single out the reasons for which they could optically manipulate microscopic particles, while the same would not have been possible with everyday objects. Indeed, a few piconewtons $(1 \mathrm{pN}=10-12 \mathrm{~N}$, for comparison the weight of an average person is around $1000 \mathrm{~N}$ ) are enough to trap a micrometer size particle. This force can be exerted by a very weak trap. Increasing the laser power larger forces can be obtained; however, an overwhelming power would be needed to trap a macroscopic object, actually high enough to destroy it.

\section{STATISTICAL PHYSICS WITH OPTICAL TWEEZERS}

\subsection{Brownian motion}

Microscopic colloidal particles immersed in water are ideal models to study statistical physics phenomena, because they undergo Brownian motion ${ }^{18,19}$. Brownian motion is the random movement of a microscopic particle immersed in a fluid and results from the collisions with the fluid molecules. Actually, Brownian motion is a very fundamental physical phenomenon in its own right, e.g., it lays at the foundations of stochastic analysis ${ }^{20}$. It was possible to familiarize the students with the concept of Brownian motion just by using the microscope they had built. The students were then encouraged to track in real-time the trajectory of a Brownian particle by drawing on a white board where the image of the particle was projected. For this purpose, a polystyrene bead was optically trapped and the CCD camera output of the microscope was projected onto the white board. Fig. 5(a) shows students charting out a particle trajectory on the white board. 

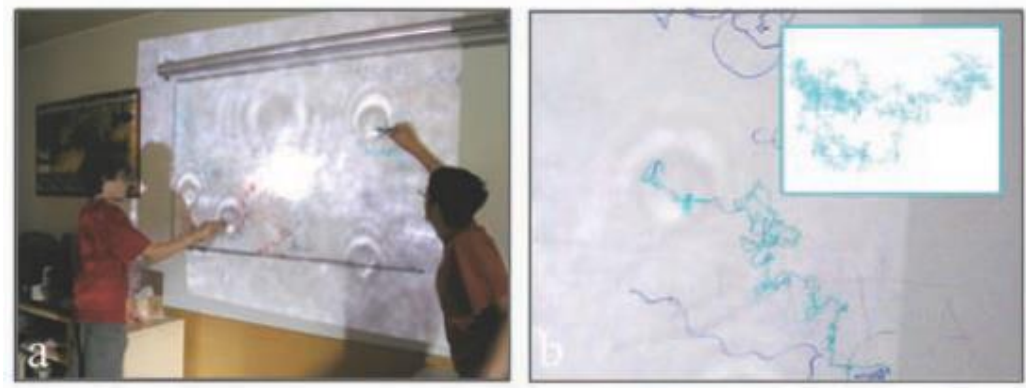

FIG. 5: Tracking Brownian motion (a) Students experiencing Brownian motion by tracking it on a screen. (b) Example of Brownian motion tracked by hand. Inset: Example of simulated Brownian motion.

A more rigorous study of the Brownian motion was obtained by analyzing the video of the particle recorded under the same conditions ${ }^{21}$. The results from the video tracking basically confirmed what the students had observed by hand. Brownian motion offers an ideal ground to introduce computer simulations to the students. It is possible to simulate Brownian motion by using a random number generator. The students employed the following simple MatLab code:

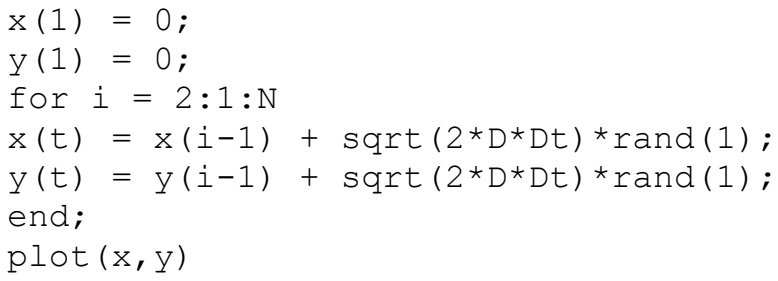

where $\mathrm{N}$ is the number of timesteps, $\mathrm{D}$ is the diffusion coefficient of the particle, $\mathrm{Dt}$ is the timestep of the simulation, and randn(1) is the MatLab function that generates a gaussian distributed random number with zero mean and unitary variance. Actually the trajectory obtained tracking the particle by hand looked quite similar to the ones that were obtained from the Brownian dynamics simulation [Fig. 5(b)], apart from the arbitrary length and time scales of the simulations.

\subsection{Drift and diffusion}

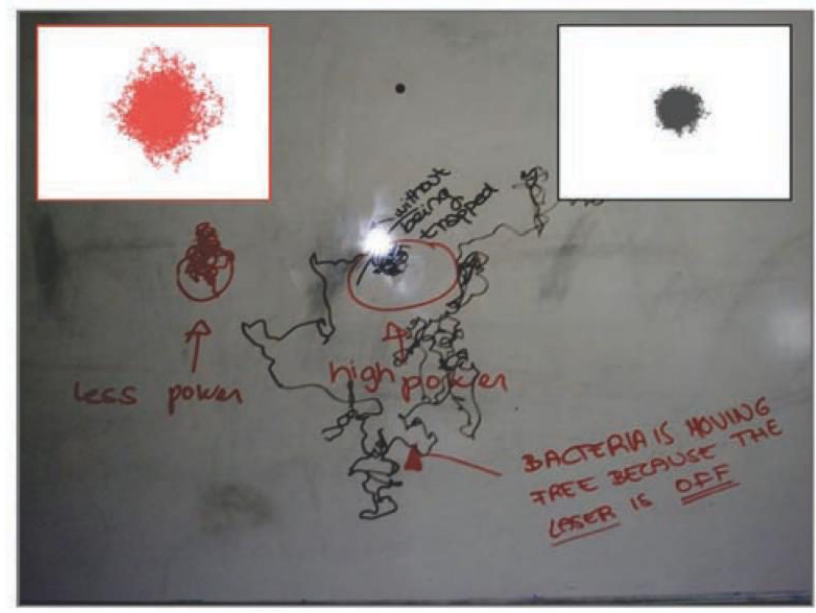

FIG. 6: Tracking Brownian motion of a trapped particle. Examples of Brownian motion tracked by hand for low (red) and high (black) laser power. Inset: Simulated Brownian motion for low (red) and high (black) stiffness. 
An optically trapped Brownian particle is also an ideal model system to discuss the interplay between the deterministic drift - due to the deterministic forces acting on the particle - and the random diffusion - due to the thermal random fluctuations produced by the hits from the water molecules - which are indeed the essential elements of any system subject to noise. The students readily recognized that an optically trapped particle stays on average at a given position. Nonetheless by plotting its trajectory, they noticed that it keeps on jiggling randomly around such position. This was a very intuitive way for the students to understand that a particle inside an optical trap undergoes a deterministic motion, which trivially keeps it in place, and a random Brownian motion.

This experiment was repeated for various laser powers [Fig. 6(a)]. It was straightforward for the students to observe that the higher the trapping power, the longer the time the particle remained trapped. This revealed that the trap strength is proportional to the optical power used for the trap ${ }^{22}$. Furthermore, the students observed that the higher the power, the smaller the region explored by the particle and the higher the frequency of its motion. The code for the simulation of the Brownian motion could be modified to account for the optical trap:

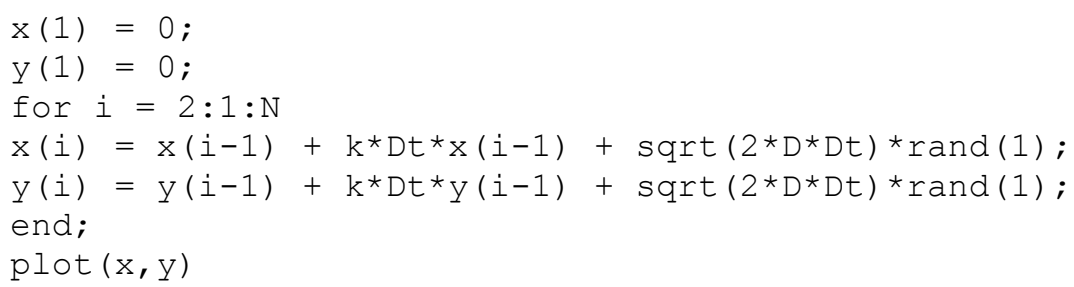

where $\mathrm{k}$ is the trap elastic constant. Fig. 6(b) shows the results of the simulations.

\subsection{Fractal nature of Brownian motion}

A fractal is a geometric shape that irrespective of how many times it is divided, each divided part looks like the original object $^{4}$. There are many examples of fractals in real life, maybe one of the best one is a brocoli. Brownian motion is a fractal two. A position time graph of a particle undergoing Brownian motion always looks very similar irrespective of the time scale in which the measurement is done. It is in this sense that Brownian motion is a fractal too. Various mathematical principles that applied to analyzing fractals can also be used to analyze Brownian motion. Therefore, from this standpoint the students were introduced to some basic concepts of fractals.

\section{BIOPHOTONICS WITH OPTICAL TWEEZERS}

\subsection{Preparation of biological specimens}
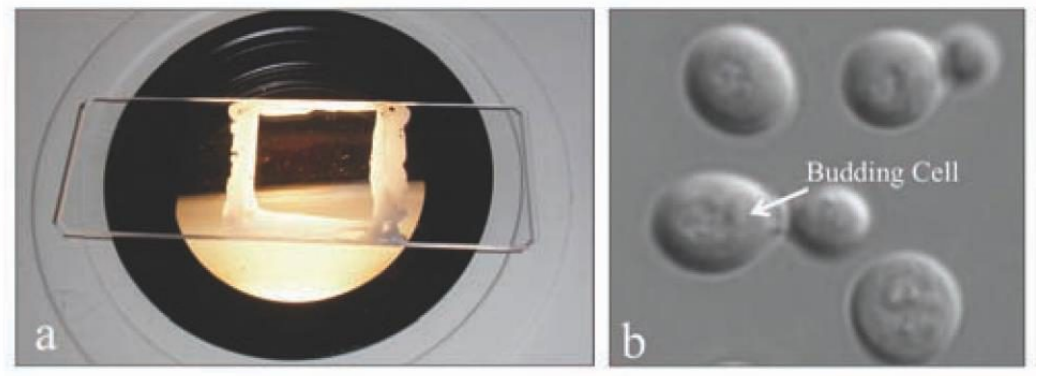

FIG. 7: Preparation of the biological specimens. (a) A specimen. (b) An image of a yeast cell.

Two biological specimens Esterichia coli (OP50) bacteria and yeast (S. cerevisiae) were employed in the biophysical experiments. Both of them are easy and safe to handle for the students. E. Coli was cultured by transferring a small amount of the bacteria into Luria Broth medium for bacterial culture and leaving them in a warm place (about $20^{\circ} \mathrm{C}$ ) overnight. For yeast cultures baker's yeast (S. cerevisiae) from the market was dissolved in a sugar solution. The 
observation samples were prepared for microscopy by mounting a small amount of the biological suspension on a glass slide and covering it with a thin coverslip. The coverslip was sealed using paraffin wax. An example of the sample is shown in Fig. 7(a).

\subsection{Study of E. Coli longevity in an optical trap}

To be optically trapped is not the natural condition for a cell: The cell is stressed and in extreme cases may even die. The students measured how long the E. Coli cells could survive in an optical trap. The strain of E. Coli used in the experiments is motile. The living cell movement can be easily distinguished from the Brownian motion of a dead cell: When the bacterium dies its motion reduces only to the Brownian component. This criterion was used to decide how long the cell had survived in the trap. The laser wavelength $(630 \mathrm{~nm})$ used was not ideal to minimize the damage to the cell. However, longevities from 5 to 30 minutes were measured. This demonstrated to the students that biological cells can be maintained alive inside an optical trap under suitable conditions.

\subsection{Budding of an yeast cell in an optical trap}

A biological process that can easily be studied in an optical trap is the budding of a yeast cell. A single yeast cell reproduces in about 2 hours through a process called budding in which a small bud appears on the mother cell, continues to increase in size, and eventually separates as a daughter cell. An optically trapped yeast cell can be maintained alive ${ }^{9}$ and undergo the budding process in an optical trap ${ }^{10}$. The students were able to observe directly the budding process inside the trap.

\section{DISCUSSION AND CONCLUSIONS}

This work demonstrates a very elegant, easy to implement and hands-on approach to introduce advanced concepts in optics, statistical physics and biophotonics to higher-secondary-school students. An optical tweezers setup that was simple yet meeting all the requirements of a standard research level apparatus was employed for the purpose. Importantly, even the construction of the tweezer itself was used as a didactic activity to introduce basic concepts in optics. Many of the concepts introduced during the summer workshop are typically taught only at universities relying on elaborate lecture sessions, which may fail to invoke the curiosity of the students. Here, optics, statistical physics and biophotonics were introduced to school students by their direct involvement in building setups and in doing very simple and intuitive experiments. The summer workshop lasted two weeks and was divided in different sections. The students were given specific tasks, e.g., building up the microscope. At the end of each session the students were encouraged to form groups and discuss. The awareness that even advanced science can be fun might motivate the students to choose science based courses at a university level and to undertake a future scientific career. We believe that the experience we report here can serve as a guideline for similar endeavors to introduce many more university level concepts to highersecondary-school students.

\section{ACKNOWLEDGMENTS}

We sincerely thank la Fundació Catalunya-La Pedrera (Barcelona, Spain) and ICFO-The Institute of Photonics Sciences (Castelldefels, Spain) for their generous financial support, which enabled us to implement the workshop. We thank the Fundació Catalunya - La Pedrera Youth in Science program for selecting our project Pick it up with light! for their educational outreach program. We thank ICONS, the ICFO student organization, for technical support. Special thanks goes to the students who participated in the two week course whose comments and insights on this program we have tried to report in the present article, and to Maria Calsamiglia and Eva Calves for their continued help. 


\section{REFERENCES}

[1] S. Chaitanya Kumar, M. Mathew, G. Volpe, O. Takayama, and A. Valencia, Education and Training in Optics and Photonics, OSA Technical Digest Series (CD) (Optical Society of America, 2009), paper ESA4.

[2] O. Takayama, A. Niederberger, P. Ghenuche, M. Mathew, and G. Volpe, Education and Training in Optics and Photonics, OSA Technical Digest Series (Optical Society of America, 2007), paper ESD6.

[3] Fundació Catalunya - La Pedrera Youth in Science program, http://www.jovesiciencia.com/.

[4] B. B. Mandelbrot, The Fractal Geometry of Nature (W. H. Freeman, New York, 1982).

[5] L. Gammaitoni, P. H•anggi, P. Jung, and F. Marchesoni, Rev. Mod. Phys. (1998).

[6] L. I. McCann, M. Dykman, and B. Golding, Nature 402, 785 (1999).

[7] G. Volpe, S. Perrone, J. M. Rubi, and D. Petrov, Phys. Rev. E 77, 051107 (2008).

[8] D. Botstein, S. A. Chervitz, and J. M. Cherry, Science 277, 1259 (1997).

[9] G. Volpe, G. P. Singh, and D. Petrov, Appl. Phys. Lett. 88, 231106 (2006).

[10] G. P. Singh, G. Volpe, C. M. Creely, H. Grotsch, I. M. Geli, and D. Petrov, J. Raman Spectrosc. 37, 858 (2006).

[11] S. P. Smith, S. R. Bhalotra, A. L. Brody, and B. L. Brown, Am. J. Phys. 67, 26 (1999).

[12] J. Bechhoefer and S. Wilson, Am. J. Phys. 70, 393 (2002).

[13] M. S. Rocha, Am. J. Phys. 77, 704 (2009).

[14] Ashkin, J. M. Dziedzic, J. E. Bjorkholm, and S. Chu, Opt. Lett. 11, 288 (1986).

[15] Ashkin, IEEE J. Sel. Top. Quant. El. 6, 841 (2000).

[16] Ashkin, Biophys. J. 61, 569 (1992).

[17] R. Di Leonardo, The trap force applet, http://glass.phys.uniroma1.it/dileonardo/Applet.php?applet=TrapForcesApplet.

[18] Babic, C. Schmitt, and C. Bechinger, Chaos 15, 026114 (2005).

[19] G. Volpe, G. Volpe, and D. Petrov, Phys. Rev. E 76, 061118 (2007).

[20] Nelson, Dynamical Theories of Brownian Motion (Springer, Berlin / Heidelberg, 1975).

[21] J. C. Crocker and D. G. Grier, J. Colloid Interfac. Sci. 179, 298 (1996).

[22] G. Volpe, J. Wehr, D. Petrov, and J. M. Rubi, J. Phys. A: Math. Theor. 42, 095005 (2009). 